\title{
A novel approach for treatment of sacrococcygeal pilonidal sinus: less is more
}

\author{
Christopher Soll • Dieter Hahnloser • Daniel Dindo • \\ Pierre-Alain Clavien • Franc Hetzer
}

Accepted: 26 July 2007 / Published online: 17 August 2007

(C) Springer-Verlag 2007

\begin{abstract}
Background The surgical management of sacrococcygeal pilonidal sinus (PS) is still a matter of discussion. Therapy ranges from complete wide excision with or without closure of the wound to excochleation of the sinus with a brush. In this paper, we introduce a novel limited excision technique. The aim of this study was to assess the morbidity and recurrence rate of this technique.

Materials and methods Limited excision consisted of a selective extirpation of the sinus after tagging the tract with methylene blue. Ninety-three consecutive patients, who underwent surgery between 2001 and 2004, were analyzed. The patients' survey was performed by mail questionnaire and telephone interview inquiring recurrence, time off work, and time to wound healing.

Results Seventy-three percent of the patients were treated in an outpatient setting. With a median follow-up of 2 years, the recurrence rate was $5 \%$. The median time off work was 2 weeks. The median wound healing time was 5 weeks. Conclusion Limited excision for PS can be done in an outpatient setting with a low recurrence rate and short time off work.
\end{abstract}

Keywords Pilonidal sinus · Pilonidal cyst · Surgery · Therapy

C. Soll $\cdot$ D. Hahnloser $(\bowtie) \cdot$ D. Dindo $\cdot$ P.-A. Clavien Department of Visceral and Transplantation Surgery, University Hospital Zuerich,

Raemistrasse 100,

CH-8091 Zuerich, Switzerland

e-mail: dieter.hahnloser@usz.ch

\section{F. Hetzer}

Department of Surgery, Cantonal Hospital,

9007 St. Gallen, Switzerland

\author{
Abbreviations \\ PS pilonidal sinus
}

\section{Introduction}

Sacrococcygeal pilonidal sinus (PS) is a common disease, but its treatment remains controversial. Surgical therapy ranges from excochleation to excisions with complex reconstructive cutaneous flaps. Among these different techniques, wide excision to the level of the sacrococcygeal fascia with open wound is probably the most performed treatment and has a recurrence rate of $5-13 \%[1,2]$. For wide excisions with primary closure of the wound such as the Limberg flap or Z-plasty, lower recurrence rates of 0 [3] and $4 \%$ [4] have been shown. However, with wide-excision techniques, most patients require hospitalization of several days $[3,5,6]$ and had a period off work up to 1 month [7].

In this study, we describe a novel technique of limited extirpation of the PS (sinusectomy). The aim of this study was to assess the morbidity and efficacy of this technique.

\section{Materials and methods}

Patient population

Ninety-three patients with a PS were operated between 2001 and 2004 in our department. The medical records were reviewed with reference to operating time, type of anesthesia, duration of hospitalization, and postoperative complications. A questionnaire was sent to all patients inquiring recurrence, wound healing time, and inability to work. Additionally, a telephone survey was performed to improve the response rate of the mail questionnaire. 
Surgical technique of limited excision (sinusectomy)

Noninfected PS was primarily treated with a limited excision according to a standardized technique: The patient was placed prone in a genupectoral position, and the shaved intergluteal fold was separated by tapes. If surgery was performed in local anesthesia, lidocaine $1 \%$ and epinephrine 1:100,000 was used. The orifices of the PS were probed, and diluted methylene blue was injected to mark the subcutaneous tract. Next, the orifices and sinus were closely excised with the scalpel or scissor following the methylene-marked tissue as shown in Fig. 1. After hemostasis, the wounds were left open to intend secondary wound healing. No postoperative antibiotic treatment was given. All patients were instructed to shower the wound at least twice a day and shave their intergluteal region once a week until complete healing was achieved. All patients were seen at least once at the outpatients' clinic and then were referred to the general practitioner.

In case of infection, PS was first drained by a small incision of the abscess in local anesthesia. Two weeks later, the sinusectomy as described above was performed.

\section{Results}

Seventy-two men (77\%) and 21 women (23\%) have been treated by sinusectomy. The median age was 28 years (range $=$

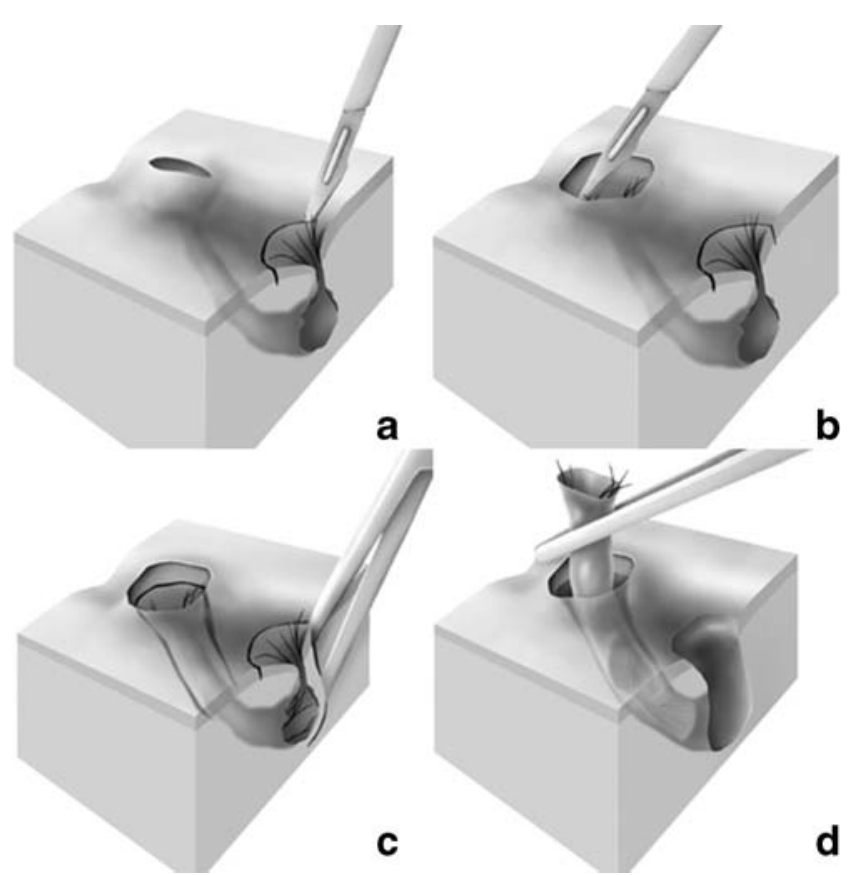

Fig. 1 Step-by-step illustration of limited excision. Close incision of pilonidal orifices after marking the PS with diluted methylene blue (a, b). Preparation and subcutaneous extirpation of the sinus (c, d)
14-47) with a median body mass index of $25.0 \mathrm{~kg} / \mathrm{m}^{2}$ $($ range $=19.4-41.0)$. Fifty-four (58\%) patients underwent primary sinusectomy. Thirty-nine $(42 \%)$ presented with an infected PS. After incision of the abscess, sinusectomy was performed 2 weeks later. None of the patients had surgery for PS before.

The sinusectomy was performed in $52 \%$ under local anesthesia. At the beginning of the study, local anesthesia was used irregularly and became the preferential type of anesthesia since 2003. The median operation time was $20 \mathrm{~min}$ (range $=20-60$ ). Seventy-three percent of patients were treated in an outpatient setting (defined as hospitalization less than $12 \mathrm{~h}$ ). The remaining $27 \%$ of patients had a median hospital stay of 1 day (range $=1-15$ ). The postoperative complication rate was $6.5 \%(n=6 / 93)$. The patients presented either with bleeding $(n=2 / 93)$, infection $(n=2 / 93)$, or delayed wound healing $(n=2 / 93)$, and all were treated conservatively. No reoperation was necessary.

Median follow-up was 28 months (range $=4-73$ ) The primary response rate to the questionnaire was $61 \%(n=57)$. After an additional telephone survey, the response rate could be improved to $81 \%(n=75)$. Eighteen patients were lost to follow-up. The median time for wound healing (defined as complete closure of the skin) after sinusectomy was 5 weeks (range $=1-30$ ). The median time off work was 2 weeks (range $=1-12)$. The recurrence rate was $5 \%(n=4 / 75)$.

\section{Discussion}

The treatment of PS is still a matter of debate. Various operations are described in the literature offering low recurrence rates but differ in morbidity, length of hospital stay, and work off time. A common sense for treatment is still lacking [8]. It is generally agreed that the treatment of choice for PS should be characterized by a short time of wound healing with a low recurrence rate, a short hospital stay, and a short time off work $[8,9]$.

For sinusectomy, we found a recurrence rate of $5 \%$ with a median follow up of 2 years. Almost three fourths of the patients $(73 \%)$ could be treated in an outpatient setting. If hospitalization was necessary, most of the patients were dismissed within 1 day. Despite a wound healing time of 5 weeks, patients returned to work within 2 weeks.

We compared our results with other studies to estimate the reliability of our data. Gupta [7] reported recurrence rates of $13 \%$ using wide-excision techniques, while other studies achieved 5\% in larger trials [2]. A recurrence rate of $3-10 \%$ is described for excision with marsupialization of the wound [10, 11]. Reconstructive flaps according to Limberg's technique come with a recurrence rate of $0-6 \%$ $[3,12]$ in prospective randomized studies. However, it was not mentioned in all these studies that surgery was per- 
formed in an outpatient setting. Hospital stay varied from 2 to 5 days $[3,7]$ using wide-excision techniques, and median time off work was reported from 19 up to 38 days [3, 13], what is considerably higher than after sinusectomy.

In our study, we found a median wound healing time of 5 weeks. Although primary closure may accelerate wound healing [1], we avoided primary closure of the wound. Many studies reported higher recurrence rates [14, 15], especially if primary closure was performed in midline [16]. However, we found not a shorter wound healing after excision and open granulation in comparative studies. A wound healing time from 7 to 12 weeks was reported [7, 11, 13].

The term of limited excision in the literature for PS is often associated with techniques according to Bascom or Lord [17, 18]. Bascom described a parallel incision a few centimetres away from the midline, where the cavity is entered and the hair and granulation tissue are curetted. Lord introduced an excochleation of the PS with a brush. Bascom's technique has been described as an outpatient procedure with a recurrence rate of $10 \%$ [19, 20]. Lord's techniques was reported with an recurrence rate of $24 \%$ and a hospital stay of 5 days [21]. Probably due to the high recurrence rate, these limited excision techniques are not performed in surgical routine.

Considering the problems of retrospective analysis, there are two issues that have to be respected when interpreting our results: First, the recurrence rate of this study might be higher as demonstrated due to a non-respondent bias. If the worst comes to the worst all non-responder $(n=18)$ might have recurrent PS. This would imply a recurrence rate of $24 \%$. Probably, within the 18 missing patients one or two of them had a recurrence. This would not markedly change the overall recurrence rate of 5-6\%. Our response rate of $81 \%$ is high compared to the mean response rate of $54 \%$ for mailed surveys [22]. This review concluded that there is not a direct relationship between response rate and bias. Surveys with high response rates may contain a nonrespondent bias, and surveys with very low response rates may not. Hence, we may assume that our findings provide a representative sample of the population.

Second, the wound healing time might have been shorter than it is reported in this study. The wound healing time was assessed by the patients themselves and was not determined by a physician. The wound healing time might more reflect the disappearance of any discomfort at the intergluteal fold than the complete closure of the skin after surgery. This may explain the wide range of the reported wound healing time ( 1 to 30 weeks). Nevertheless, after 2 weeks, the wounds were nearly asymptomatic, as most patients fully returned to work.

In conclusion, sinusectomy is a novel limited excision technique for non-infected PS. It may be performed in an outpatient setting and under local anaesthesia with low morbidity and low recurrence rate. Time off work is considerably short. Therefore sinusectomy is a promising novel approach in the treatment of PS.

\section{References}

1. Spivak H, Brooks VL, Nussbaum M, Friedman I (1996) Treatment of chronic pilonidal disease. Dis Colon Rectum 39:1136-1139

2. Sondenaa K, Nesvik I, Andersen E, Soreide JA (1996) Recurrent pilonidal sinus after excision with closed or open treatment: final result of a randomised trial. Eur J Surg 162:237-240

3. Akca T, Colak T, Ustunsoy B, Kanik A, Aydin S (2005) Randomized clinical trial comparing primary closure with the Limberg flap in the treatment of primary sacrococcygeal pilonidal disease. Br J Surg 92:1081-1084

4. Fazeli MS, Adel MG, Lebaschi AH (2006) Comparison of outcomes in Z-plasty and delayed healing by secondary intention of the wound After excision of the sacral pilonidal sinus: results of a randomized, clinical trial. Dis Colon Rectum 49:1831-1836

5. Topgul K, Ozdemir E, Kilic K, Gokbayir H, Ferahkose Z (2003) Long-term results of Limberg flap procedure for treatment of pilonidal sinus: a report of 200 cases. Dis Colon Rectum 46: $1545-1548$

6. Aydede H, Erhan Y, Sakarya A, Kumkumoglu Y (2001) Comparison of three methods in surgical treatment of pilonidal disease. ANZ J Surg 71:362-364

7. Gupta PJ (2005) Comparative study between radiofrequency sinus excision and open excision in sacro-coccygeal pilonidal sinus disease. Dig Surg 22:459-463

8. Chintapatla S, Safarani N, Kumar S, Haboubi N (2003) Sacrococcygeal pilonidal sinus: historical review, pathological insight and surgical options. Tech Coloproctology 7:3-8

9. Sharma PP (2006) Multiple Z-plasty in pilonidal sinus - a new technique under local anesthesia. World J Surg 30:2261-2265

10. Oncel M, Kurt N, Kement M, Colak E, Eser M, Uzun H (2002) Excision and marsupialization versus sinus excision for the treatment of limited chronic pilonidal disease: a prospective, randomized trial. Tech Coloproctol 6:165-169

11. Gencosmanoglu R, Inceoglu R (2005) Modified lay-open (incision, curettage, partial lateral wall excision and marsupialization) versus total excision with primary closure in the treatment of chronic sacrococcygeal pilonidal sinus: a prospective, randomized clinical trial with a complete two-year follow-up. Int J Colorectal Dis 20:415-422

12. Cihan A, Ucan BH, Comert M, Cesur A, Cakmak GK, Tascilar O (2006) Superiority of asymmetric modified Limberg flap for surgical treatment of pilonidal disease. Dis Colon Rectum 49: 244-249

13. Perruchoud C, Vuilleumier H, Givel JC (2002) Pilonidal sinus: how to choose between excision and open granulation versus excision and primary closure? Study of a series of 141 patients operated on from 1991 to 1995 . Swiss Surg 8:255-258

14. Kronborg O, Christensen K, Zimmermann-Nielsen C (1985) Chronic pilonidal disease: a randomized trial with a complete 3-year follow-up. Br J Surg 72:303-304

15. al-Hassan HK, Francis IM, Neglen P (1990) Primary closure or secondary granulation after excision of pilonidal sinus? Acta Chir Scand 156:695-699

16. Petersen S, Koch R, Stelzner S, Wendlandt TP, Ludwig K (2002) Primary closure techniques in chronic pilonidal sinus: a survey of 
the results of different surgical approaches. Dis Colon Rectum 45: $1458-1467$

17. Lord PH, Millar DM (1965) Pilonidal sinus: a simple treatment. Br J Surg 52:298-300

18. Bascom J (1980) Pilonidal disease: origin from follicles of hairs and results of follicle removal as treatment. Surgery 87:567-572

19. Parvaiz A, Kennedy R (2001) Bascom's procedure in the daysurgical management of symptomatic pilonidal sinus. Br J Surg $88: 155-156$
20. Senapati A, Cripps NP, Thompson MR (2000) Bascom's operation in the day-surgical management of symptomatic pilonidal sinus. Br J Surg 87:1067-1070

21. Muller XM, Rothenbuhler JM, Frede KE (1992) [Sacrococcygeal cyst: surgical techniques and results]. Helv Chir Acta 58:889-892

22. Asch DA, Jedrziewski MK, Christakis NA (1997) Response rates to mail surveys published in medical journals. J Clin Epidemiol 50:1129-1136 